patient information document was produced for prolonged jaundice as no current one existed. Furthermore, parent experience was recorded during the introduction of the clinics to ensure they remained satisfied with the service delivered.

The new junior doctor-led clinics started in June 2017. Preliminary data has shown the wait time for the paediatric rapid access clinic has already reduced to below 2 days on average for June - August 2017. This compares to 3.5 days on average for the same period in 2016.

The introduction of this new junior doctor-led clinic for prolonged jaundice babies continues to provide a high-level service for patients and their parents, whilst reducing the wait time for the consultant-led rapid access clinic to the internal standard of less than 2 days on average.

\section{G385(P) A SAFETY REVOLUTION: CROSS-SPECIALITY ENGAGEMENT OF JUNIOR DOCTORS IN PATIENT SAFETY}

BP Marlow, N Nathwani. Paediatrics, Luton and Dunstable University Hospital, Luton, UK

\subsection{6/archdischild-2018-rcpch.374}

Problem The current obstacles to paediatric junior doctors engaging in patient safety include poor compliance with the safety reporting process (Datix - only 2\% completed by doctors locally) and lack of understanding in what the process is trying to achieve. There is also a 'fear of failure culture' so doctors are more likely not to admit to mistakes. High turnover of staff leads to problems with team dynamics and lack of 'buy in' into the departmental safety culture.

Aims The aim is to foster an open safety culture, remove this fear of blame mentality and promote collective learning from error. I want to double the number of Datix reported by junior doctors within 6 months.

Methods The interventions are going to be: departmental induction, monthly clinical governance meetings, and interprofessional simulation. Outcomes are going to be measured through a pre and post placement questionnaire and the numbers of Datix logged by paediatric junior doctors six months pre and six months post-intervention.

Results $100 \%$ of doctors stated patient safety was 'important' or 'very important' to clinical care. $100 \%$ knew what a Datix was, but only $16 \%$ had filled one in during the previous six months. This was reflected in only 5 out of 139 Datix (3.6\%) being filled in by junior doctors. After the planned interventions Datix completion by paediatric junior doctors improved to $10 / 126(8.1 \%)$ - over a 2 fold increase from the previous six months.

The aim for the next 12 months is to expand this safety engagement across the whole hospital establishing a 'safety board' to which an elected trainee from each speciality will be a part and establish a monthly grand round looking at a clinical incident or datix to promote an open 'no blame' approach that will become part of the hospital culture.

\section{G386(P) COMBINING QUALITY IMPROVEMENT AND HUMAN FACTOR TRAINING TO EFFECT SUSTAINABLE CHANGE}

${ }^{1,2} \mathrm{~K}$ Pryde, ${ }^{1} \mathrm{~A}$ Robson, ${ }^{1} \mathrm{~K}$ Sykes. 'Child Health, Southampton Children's Hospital, Southampton, UK; ${ }^{2}$ School for Quality Improvement, Health Education England, Wessex, UK

\subsection{6/archdischild-2018-rcpch.375}

Background There is evidence to suggest that safety interventions combining quality improvement and team work (systems) training are more effective than those adopting either approach alone. ${ }^{1}$ This has important implications for safety and quality improvement (QI) strategies in hospitals. We piloted an initiative combining these factors as a potential model for cascading QI training. We chose the PICU team who already have an annual programme of team training days.

Methods A one day programme was designed consisting of an overview of basic QI methods, an introduction to human factors, and followed by an interactive team treasure hunt with a final session discussing ideas for improvement on PICU. All staff members from domestic to clinical lead, including visiting teams such as psychology and pharmacy were invited to attend. All the improvement ideas were displayed in the staff coffee room and through 'dot-voting' the staff agreed which projects to pursue. Each project identified a nursing and clinical lead from the unit. A combination of facilitated learning sessions on QI tools and techniques appropriate to the projects needs and mentoring was used to support the improvement projects in practice.

Results Approximately 100 staff attended the training days which generated over 20 ideas for improvement.

Two projects have been pursued more actively over a year improving discharge prior to $3 \mathrm{pm}$ and debriefing after significant events. Each has engaged a range of professionals from a spectrum of seniority from the unit. The considerable success of these individual projects is shared separately.

Conclusions This pilot has shown that it is possible to deliver generic training in QI methods and human factors as well as specific improvements through a dedicated project. This has been achieved with no additional time other than the first team training day which was already part of the education programme. The quality improvement methodology and human factor skills are transferrable and have increased the capability of the units' staff to approach future improvement work.

We propose this is a cost effective method of effecting real change as well as training in both quality improvement methods and human factors which others should explore adopting.

\section{REFERENCE}

1. McCulloch P, Morgan $L$, New $S$, et al. Combining systems and teamwork approaches to enhance the effectiveness of safety improvement interventions in surgery: The safer delivery of surgical services (S3) program. Ann Surg. Ann Surg 2017;265:90-96. 